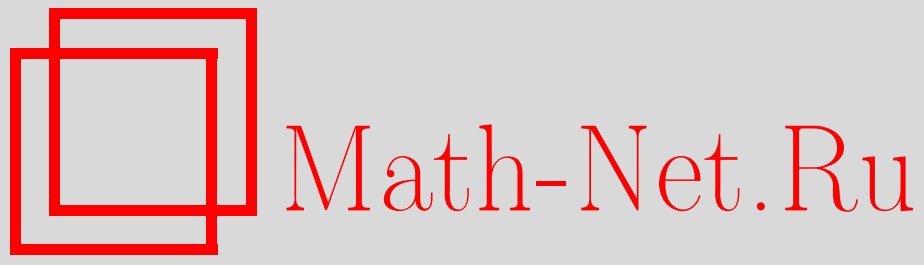

А. В. Стояновский, Описание гладких векторов представления Вейля в геометрической реализации, Функи. анализ и его прил., 2006, том 40, выпуск $3,90-93$

DOI: https://doi.org/10.4213/faa750

Использование Общероссийского математического портала Math-Net.Ru подразумевает, что вы прочитали и согласны с пользовательским соглашением

http://www . mathnet.ru/rus/agreement

Параметры загрузки:

IP: 54.174.149.18

26 апреля 2023 г., 12:06:58

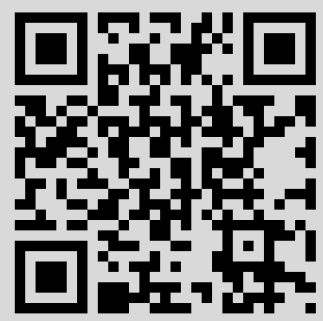


УДК 512.815

\title{
Описание гладких векторов представления Вейля в геометрической реализации*
}

\author{
(c) 2006. А. В. Стояновский
}

1. Основным результатом заметки является описание образа представления Вейля ([1]-[3]) двулистного накрытия симплектической группы $\operatorname{Sp}(2 n, \mathbb{R})$ в пространстве Шварца $S=S\left(\mathbb{R}^{n}\right)$ комплекснозначных функций $\psi\left(x_{1}, \ldots, x_{n}\right)$ и в пространстве обобщенных функций $S^{\prime}=S^{\prime}\left(\mathbb{R}^{n}\right)$ при интегральном преобразовании, переводящем функцию $\psi$ в функцию

$$
u\left(Z_{j k}\right)=u_{\psi}\left(Z_{j k}\right)=\int \overline{\psi\left(x_{1}, \ldots, x_{n}\right)} e^{\frac{i}{2} \sum_{j, k} Z_{j k} x_{j} x_{k}} d x_{1} \cdots d x_{n}
$$

на верхней полуплоскости Зигеля $\mathscr{S} \mathscr{G}$, т. е. на пространстве симметричных комплексных матриц $Z=\left(Z_{j k}\right)$ с положительно определенной мнимой частью.

Автор признателен В. В. Долотину и Ю. А. Неретину за многочисленные полезные обсуждения.

2. Определения и обозначения. Пусть $S_{+}, S_{+}^{\prime}\left(S_{-}, S_{-}^{\prime}\right)-$ подпредставления четных (соответственно нечетных) функций в пространствах $S, S^{\prime}$.

Операторы $\partial / \partial Z_{j k}$ для любых $j, k$ определяются из равенств

$$
d u=\sum_{j, k} \frac{\partial}{\partial Z_{j k}} u d Z_{j k}=\sum_{j<k} 2 \frac{\partial}{\partial Z_{j k}} u d Z_{j k}+\sum_{j} \frac{\partial}{\partial Z_{j j}} u d Z_{j j} .
$$

Пусть $\overline{\mathscr{S} \mathscr{G}}$ - замыкание верхней полуплоскости $\mathscr{S} \mathscr{G}$ при ее естественном вложении в комплексный лагранжев грассманиан. Пусть $\operatorname{Mp}(2 n, \mathbb{R})$ - метаплектическая группа (двулистное накрытие группы $\operatorname{Sp}(2 n, \mathbb{R})$ ), состоящая из пар

$$
\left(\left(\begin{array}{ll}
A & B \\
C & D
\end{array}\right), \sqrt{\operatorname{det}(C Z+D)}\right)
$$

где $\left(\begin{array}{cc}A & B \\ C & D\end{array}\right) \in \operatorname{Sp}(2 n, \mathbb{R})(A, B, C, D$ суть $n \times n$-матрицы; группа $\operatorname{Sp}(2 n, \mathbb{R})$ coxpaняет симплектическую форму с матрицей $\left.\left(\begin{array}{cc}0 & E \\ -E & 0\end{array}\right)\right)$, a $\sqrt{\operatorname{det}(C Z+D)}$ есть одна из двух непрерывных ветвей квадратного корня из $\operatorname{det}(C Z+D) \neq 0, Z \in \mathscr{S} \mathscr{G}$. Группа $\operatorname{Sp}(2 n, \mathbb{R})$ действует на верхней полуплоскости Зигеля по формуле

$$
Z \mapsto(A Z+B)(C Z+D)^{-1} .
$$

Пусть $\mu$ - естественное $\operatorname{Mp}(2 n, \mathbb{R})$-эквивариантное комплексное линейное расслоение над $\overline{\mathscr{S} \mathscr{G}}$, индуцированное вложением замыкания $\overline{\mathscr{S} \mathscr{G}}$ в проективизацию пространства $S^{\prime}$, определяемым следующим образом. Лагранжево подпространство $L \in \overline{\mathscr{S} \mathscr{G}}$ переходит в функцию $\psi_{L} \in S^{\prime}$, определяемую однозначно с точностью до пропорциональности как решение системы уравнений

$$
\sum_{j}\left(v_{j k} x_{j}+w_{j k} i \frac{\partial}{\partial x_{j}}\right) \psi_{L}=0, \quad 1 \leqslant k \leqslant n
$$

*Работа выполнена при частичной поддержке гранта РФФИ № 04-01-00640. 
где векторы $\left(v_{j k}, w_{j k}\right)_{j=1}^{n}$ составляют произвольный базис в $L$. Расслоение $\mu$ можно охарактеризовать тривиализациями и функциями перехода. А именно, над полуплоскостью $\mathscr{S} \mathscr{G}$ расслоение тривиально (можно выбрать представитель $\psi_{L}=\exp \left(\frac{i}{2} \sum Z_{j k} x_{j} x_{k}\right)$ для $L$, соответствующего $\left.Z\right)$, а действию элемента $(2)$ группы $\operatorname{Mp}(2 n, \mathbb{R})$ соответствует функция перехода $1 / \sqrt{\operatorname{det}(C Z+D)}$. Ограничение расслоения $\mu$ на вещественный лагранжев грассманиан есть тензорное произведение расслоения полуплотностей и расслоения Маслова (см. [3, $\S 21.6])$.

Пусть $W$ - тавтологическое комплексное векторное расслоение ранга $n$ над $\overline{\mathscr{S} \mathscr{G}}$, слой которого над точкой $L$ есть само пространство $L$.

Интегральное преобразование (1) переводит нечетные функции в 0. Для нечетных функций $\psi$ определяется следующий аналог этого преобразования:

$$
u_{l}(Z)=u_{l, \psi}(Z)=\int \overline{\psi\left(x_{1}, \ldots, x_{n}\right)} x_{l} e^{\frac{i}{2} \sum Z_{j k} x_{j} x_{k}} d x_{1} \cdots d x_{n}, \quad 1 \leqslant l \leqslant n .
$$

\section{3. Теоремы.}

ТЕорема 1. Преобразование (1) отождествляет пространство $S_{+}$с пространством голоморфных функиий $u(Z)$, удовлетворяющих уравнениям

$$
\frac{\partial}{\partial Z_{j l}} \frac{\partial}{\partial Z_{k m}} u=\frac{\partial}{\partial Z_{j m}} \frac{\partial}{\partial Z_{k l}} u
$$

и продолжающихся до сечений расслоения $\mu^{*}$ на замыкании верхней полуплоскости Зигеля, непрерывных вместе со всеми производными по действию алгебры Ли группъ $\operatorname{Sp}(2 n, \mathbb{R})$.

Теорема 2. Преобразование (3) отождествляет пространство $S_{-}$с пространством голоморфных вектор-функиий $\left(u_{l}(Z)\right)$, удовлетворяющих уравнениям

$$
\frac{\partial}{\partial Z_{j k}} u_{l}(Z)=\frac{\partial}{\partial Z_{j l}} u_{k}(Z)
$$

и продолжающихся до сечений расслоения $W \otimes \mu^{*}$ на замыкании верхней полуплоскости Зигеля, непрерывных вместе со всеми производными по действию алгебры Ли группь $\operatorname{Sp}(2 n, \mathbb{R})$.

Следующая теорема была сформулирована в качестве гипотезы Ю. А. Неретиным.

ТЕорема 3. Преобразование (1) отождествляет пространство $S_{+}^{\prime}$ с пространством голоморфных функиий $u(Z)$, которые удовлетворяют уравнениям (4) и имеют полиномиальный рост вблизи гранищы верхней полуплоскости, точнее, удовлетворяют оченке

$$
|u(Z)| \leqslant C(1+|Z|)^{M}\left(1+|\operatorname{det}(\operatorname{Im} Z)|^{-1}\right)^{N}
$$

для некоторьх $C, M, N$. Здесь $|Z|$ есть любая норма на пространстве матрии.

ТЕорема 4. Преобразование (3) отождествляет пространство $S_{-}^{\prime}$ с пространством голоморфных вектор-функиий $\left(u_{l}(Z)\right)$, компоненты которых удовлетворяют уравнениям (5) и оценке (6). 
Теорема 5. При отождествлении из теорем 1, 3 элемент, обратный элементу (2) группь $\mathrm{Mp}(2 n, \mathbb{R})$, переводит функиию и $Z)$ в функиию

$$
v(Z)=\frac{1}{\sqrt{\operatorname{det}(C Z+D)}} u\left((A Z+B)(C Z+D)^{-1}\right) .
$$

При отождествлении из теорем 2, 4 этот же элемент переводит векторфункиию $\left(u_{l}(Z)\right)$ в функиию $\left(v_{l}(Z)\right)$, где

$$
\sum_{l}(C Z+D)_{l}^{k} v_{l}(Z)=\frac{1}{\sqrt{\operatorname{det}(C Z+D)}} u_{k}\left((A Z+B)(C Z+D)^{-1}\right) .
$$

4. Идеи доказательств. Доказательство теоремы 3 основано на том, что преобразование (1) для обобщенной функции $\psi$ есть композиция прямого образа при отображении

$$
x \mapsto b, \quad b=\left(b_{j k}\right), \quad b_{j k}=x_{j} x_{k},
$$

и преобразования Фурье-Лапласа. Нужно описать образ этих преобразований, что делается стандартными средствами теории обобщенных функций.

Теоремы 4 и 2 легко следуют из теорем 3 и 1. Теорема 5 очевидна.

Существенно доказать теорему 1 . Для этого $L_{2}$-норма функции $\psi \in S_{+}$выражается через функцию $u_{\psi}(Z)$ как некоторый интеграл от ее граничных значений на вещественном лагранжевом грассманиане. Затем показывается, что для произвольной функции $u(Z)$, удовлетворяющей условиям теоремы, этот интеграл конечен. Согласно теореме $3, u=u_{\psi}$ для некоторого $\psi \in S_{+}^{\prime}$. Отсюда выводится, что $\psi \in L_{2}$. Аналогично с использованием $\operatorname{Mp}(2 n, \mathbb{R})$-эквивариантности доказывается, что $x^{\alpha} \partial^{\beta} \psi / \partial x^{\beta} \in L_{2}$ для произвольных мультииндексов $\alpha, \beta$. Отсюда следует, что $\psi \in S_{+}$.

5. Замечания. 1. Доказательства теорем не дают описания образа пространства $L_{2}$ при преобразованиях (1), (3). Для нахождения этого образа желательно найти инвариантную формулу, выражающую $L_{2}$-норму функции $\psi$ через функцию $u_{\psi}(Z)$. Формально такую формулу написать нетрудно:

$$
\text { const } \cdot \int_{\mathscr{S} \mathscr{G}} u(Z) \overline{u(Z)} \frac{d Z \overline{d Z}}{(\operatorname{det} \operatorname{Im} Z)^{n+1 / 2}} .
$$

Сложнее придать ей смысл. По-видимому, это нужно делать аналитическим продолжением по показателю $n+1 / 2$. По этому поводу ср. [4].

2. Группа $\mathrm{Mp}(2 n, \mathbb{R})$ действует еще в пространстве Гельфанда-Шилова $S_{1 / 2}^{1 / 2}\left(\mathbb{R}^{n}\right)[5]$ и в сопряженном пространстве. Интересно было бы найти образ этих пространств при преобразованиях (1), (3).

\section{ЛитератУРА}

[1] Ж. Лион, М. Вернь, Представление Вейля, индекс Маслова и тэта-рлды, Мир, M., 1983. [2] Yu. A. Neretin, in: Contemporary Mathematical Physics, Transl. Amer. Math. Soc. Ser. 2, vol. 175, 1996, 37-135. [3] Л. Хёрмандер, Анализ линейных дифференииальных операторов с частными производными, т. 3, Мир, М., 1987.

[4] Yu. A. Neretin, 
http://arxiv.org/math.RT/0012220. [5] И. М. Гельфанд, Г. Е. Шилов, Обобщенные функции. Bып. 2. Пространства основных и обобщенных фбункций, Физматгиз, М., 1958.

Московский центр непрерывного

Поступило в редакцию математического образования

e-mail: stoyan@mccme.ru

8 февраля 2005 г.

УДК 517.98

\title{
Аддитивность гомологических размерностей для некоторого класса банаховых алгебр*
}

\author{
(c) 2006. С. Б. ТАБАлдЫЕВ
}

В 1997 г. Ю. В. Селиванов в [1] доказал следующие «формулы аддитивности» для гомологических размерностей (определения см. ниже): если $A-y н u-$ тализация бипроективной коммутативной банаховой алгебры с бесконечным спектром, а $B$ - произвольная унитальная банахова алгебра, то $\operatorname{dg} A \widehat{\otimes} B=$ $\operatorname{dg} A+\operatorname{dg} B u \operatorname{db} A \widehat{\otimes} B=\operatorname{db} A+\operatorname{db} B$.

Пусть $\Omega$ - бесконечное метризуемое компактное топологическое пространство, у которого производное множество некоторого конечного порядка пусто. (Напомним [2], что производное множество топологического пространства есть множество всех его предельных точек.) Пусть $A=C(\Omega)$ - банахова алгебра всех непрерывных функций на $\Omega$ с равномерной нормой. Мы утверждаем (в теореме 2), что формулы аддитивности верны и в этом (новом) случае; легко убедиться в том, что если $C(\Omega)$ - унитализация бипроективной алгебры, то уже его производное множество первого порядка $\Omega^{\prime}$ состоит из одного элемента, а производное множество второго порядка $\Omega^{\prime \prime}=\left(\Omega^{\prime}\right)^{\prime}$ пусто.

Пусть $X$ - левый банахов $A$-модуль, а $E$ - банахово пространство. Тогда тензорное произведение $X \widehat{\otimes} E$ является левым банаховым $A$-модулем относительно внешнего умножения, определяемого формулой $a \cdot(x \otimes u)=(a \cdot x) \otimes u$ для $a \in A, x \in X$ и $u \in E$. (Определение проективного тензорного произведения $\widehat{\otimes}$ банаховых пространств и проективного тензорного произведения $\widehat{\otimes}_{A}$ левого и правого банаховых модулей над банаховой алгеброй $A$ можно найти, например, в книге [3].)

Предположим, что $A$ - унитальная банахова алгебра, а $X$ - унитальный левый банахов $A$-модуль. Напомним, что модуль $X$ называется проективным (см. [3]), если он является прямым слагаемым $A$-модуля $A \widehat{\otimes} E$ для некоторого банахова пространства $E$. Аналогично определяется проективный унитальный банахов $A$-бимодуль. Напомним также, что длина самой короткой проективной допустимой (т. е. расщепимой как комплекс банаховых пространств) резольвенты $A$-модуля $X$ называется его гомологической размерностью и обозначается через $A$ dh $X$. Гомологическая размерность банахова $A$-бимодуля определяется аналогично. Верхняя грань гомологических размерностей унитальных левых

*Работа выполнена при финансовой поддержке Российского фонда фундаментальных исследований (грант 05-01-00982). 